\title{
QUEEN'S
UNIVERSITY
BELFAST
}

\section{How do women feel about being weighed during pregnancy? A qualitative exploration of the opinions and experiences of postnatal women}

Allen-Walker, V., Mullaney, L., Turner, M. J., Woodside, J. V., Holmes, V. A., McCartney, D. MA., \& McKinley, M. C. (2016). How do women feel about being weighed during pregnancy? A qualitative exploration of the opinions and experiences of postnatal women. Midwifery. https://doi.org/10.1016/j.midw.2016.12.006

Published in:

Midwifery

Document Version:

Peer reviewed version

Queen's University Belfast - Research Portal:

Link to publication record in Queen's University Belfast Research Portal

Publisher rights

(C) 2016 Published by Elsevier Ltd.

This manuscript version is made available under the CC-BY-NC-ND 4.0 license http://creativecommons.org/licenses/by-nc-nd/4.0/,which permits distribution and reproduction for non-commercial purposes, provided the author and source are cited.

\section{General rights}

Copyright for the publications made accessible via the Queen's University Belfast Research Portal is retained by the author(s) and / or other copyright owners and it is a condition of accessing these publications that users recognise and abide by the legal requirements associated with these rights.

Take down policy

The Research Portal is Queen's institutional repository that provides access to Queen's research output. Every effort has been made to ensure that content in the Research Portal does not infringe any person's rights, or applicable UK laws. If you discover content in the Research Portal that you believe breaches copyright or violates any law, please contact openaccess@qub.ac.uk. 


\section{Abstract \\ Objective}

To explore routine weighing in antenatal care and weight management in pregnancy with women who have been weighed during pregnancy.

\section{Design}

A qualitative study utilising semi-structured telephone interviews, and thematic analysis.

\section{Setting}

Participants resided in Dublin, Ireland and had been weighed during pregnancy.

\section{Participants}

Individual telephone interviews conducted with ten postpartum women (nine months postpartum).

\section{Findings}

Experiences of routine weighing were positive, and participants believed it should be part of standard antenatal care. Several benefits to routine weighing were cited, including providing reassurance and minimising postpartum weight retention. It was felt that there was a lack of information provided on gestational weight gain and healthy lifestyle in pregnancy, and that healthcare professionals are ideally placed to provide this advice. Increased information provision was seen as a method to improve healthy lifestyle behaviours in pregnancy.

\section{Key conclusions and implications for practice}

These findings contribute to the current debate about the re-introduction of routine weighing throughout pregnancy (Allen-Walker et al. 2016). Women stated that they expected to be weighed during pregnancy and, contrary to previous claims, there was no evidence that routine weighing during antenatal care caused anxiety. From 
discussions it was clear that women desired more information on gestational weight gain and a healthy lifestyle, and felt that health professionals should provide this.

\section{Keywords}

Weighing, pregnancy, opinions, gestational weight gain, qualitative.

Funding: This work was supported by a Department of Education and Learning, Northern Ireland, PhD studentship award.

\section{Introduction}

The adverse outcomes associated with overweight and obesity in pregnancy, for mothers, their infants and the healthcare professionals caring for them, are well documented. Pregnant mothers who are overweight or obese are at higher risk of gestational diabetes mellitus, caesarean section, postpartum haemorrhage (Scott-Pillai et al. 2013), pre-eclampsia and maternal death (National Institute for Clinical Excellence, 2010). Mothers who are obese are more likely to experience miscarriage and stillbirth (Modder and Fitzsimons, 2010), and greater pre-pregnancy maternal weight is associated with increased offspring adiposity (Fraser et al. 2010). Furthermore, postpartum weight retention and excess gestational weight gain are established predictors of long term obesity (Leslie et al. 2013). The impact on the healthcare system is significant; caring for pregnant women with obesity often requires the input of several specialities and additional equipment (Heslehurst et al. 2007), and reports of physical injury to individual health professionals as a consequence of supporting mothers with obesity in labour have been made (KnightAgarwal et al. 2014).

Encouraging appropriate weight gain during pregnancy is a key factor that will influence a woman's weight trajectory and health status later in life. However, there 
are currently no safe ranges of GWG in the UK owing to a lack of evidence to underpin such guidance (National Institute for Clinical Excellence, 2010). As discussed in a recent commentary, beginning to weigh women during pregnancy would help to provide the evidence needed to fill this gap and may also have additional benefits in relation to antenatal care (Allen-Walker et al. 2016). Weighing women throughout pregnancy is no longer standard practice in the UK, it was phased out in the 1990s due to arguments that weight at booking was a more sensitive predictor of infant outcomes, and that frequent weighing caused unnecessary anxiety in women (Dawes and Grudzinskas, 1991).

Reconsidering weighing throughout pregnancy would be one approach to informing the evidence base regarding appropriate gestational weight gain ranges for women in the UK, however there are many factors to consider before introducing such a practice, not least, considering the views of women.

Some qualitative research to date provides insights into factors which may influence the feasibility of re-introducing routine weighing during pregnancy. From the viewpoint of health professionals, qualitative research exploring the experiences of midwives caring for obese women in labour has found that midwives are embarrassed about raising the issue of obesity and are concerned about broaching the topic without causing offence (Heslehurst et al. 2011, Singleton and Furber 2014). Health professionals have reported a lack of knowledge and opportunity to discuss gestational weight gain with women, as well as lacking the opportunity to monitor weight due to guidelines to only weigh at booking (Olander et al. 2011). Focus groups findings from midwifery health professionals in Australia reflect ongoing concerns about how to introduce the sensitive topic of weight with pregnant 
women, and that not routinely weighing may suggest to women that GWG doesn't matter (Schmied et al. 2011). A more recent Australian study with obstetricians and midwives raised the point that weighing and discussing weight gain are 'out of fashion', although participants in this study felt weighing all women would prevent judgemental attitudes (as opposed to targeting obese women), and would be useful in considering future health outcomes of women (Knight-Agarwal et al. 2014). A qualitative study considering how to develop maternity services to address obesity, carried out with maternity unit staff in the North East of England, elicited a range of views on the use of weight monitoring in pregnancy, from feeling there may be little point in monitoring the weight of mothers who are already obese, to thinking there is little point in giving advice if weight is not monitored as well (Heslehurst et al. 2011). These studies all highlight that health professionals feel GWG is important to address, yet fear offending women and do not always know how to approach the topic.

Two recent studies looking at routine weighing as a method of minimising gestational weight gain also provide insight into the acceptability of routine weighing to women and health professionals. As part of a feasibility study of regular weighing, accompanied by weight gain targets set by community midwives in England, midwives and pregnant women were interviewed about their views of the intervention (Daley et al. 2015). Women felt motivated to think about their weight gain in terms of eating and physical activity habits as a result of being weighed, and responded positively to the importance of being weighed routinely. The midwives reported no issues in incorporating routine weighing into the appointment, and perceived women were happy to be weighed (Daley et al. 2015). Similarly, women allocated to the intervention arm of a randomised controlled trial in Australia, comparing routine 
weighing to usual care, were asked to complete questionnaires about their experiences of being weighed. The majority (73\%) reported being very satisfied with being weighed, and likewise $73 \%$ reported being not at all anxious as a result of being weighed, indicating routine weighing as part of antenatal care is acceptable to women (Brownfoot et al. 2016).

Whilst these studies highlight important considerations to reintroducing routine weighing, most focus on experiences of caring for obese patients specifically, and have been undertaken in Australia and England. Findings emerging from intervention studies which aim to prevent excess gestational weight gain, on the acceptability of weighing to women, also provide valuable insight into issues which need to be taken into account. However the study presented here provides a more in-depth consideration of the experience of routine weighing, by women who have recently delivered. This allows exploration into the potential influences of regular weighing on the postpartum period, as well as during pregnancy itself, thus providing new insights to help inform the debate about the merits or otherwise of reintroducing the practice to routine care.

\section{Study rationale}

This study involved interviewing postnatal women who were weighed throughout their pregnancies as part of a research project. Since women are not routinely weighed during their pregnancies in the UK and Ireland, this provided a unique opportunity to explore how the women felt about being weighed, and to consider their opinions more generally on weight management in pregnancy. This study approach, as well as the specific issues that were explored, provides a more 
comprehensive qualitative view on the issue of routine weighing and weight management during pregnancy, than is currently available.

\section{Research aims}

The aim of this study was to explore the attitudes and opinions of postnatal women, who had been weighed during their most recent pregnancy, towards:

- Expectations regarding weight gain in pregnancy

- Their experiences of being weighed throughout pregnancy

- Weight management and pregnancy

\section{Method}

\section{Study design}

A qualitative methodology design was employed in order to elicit an in-depth account of the participants' lived experiences of the topic matter (Braun and Clarke, 2006); the qualitative paradigm has been successfully employed to explore women's experiences of obesity and pregnancy (Olander et al., 2011; Atkinson et al., 2013). Since this study was conducted with post-partum women, telephone interviews were chosen as the most appropriate method to maximise likelihood of participation. Attending focus groups or face-to-face interviews presents particular logistical challenges which are likely to be off-putting for this target group.Semi-structured telephone interviews were used to collect data, analysed using thematic analysis described below.

\section{Recruitment and sample}


Participants were recruited from the Body Composition in Pregnancy (BCIP) study, at Coombe Women and Infants Hospital in Dublin. The Coombe Hospital in Dublin is one of the largest maternity hospitals in the European Union and cares for women from all socio-economic groups and from across the urban-rural divide. Women were approached to take part in the BCIP study after an ultrasound scan confirmed an ongoing singleton pregnancy (Mullaney et al., 2016b). The BCIP study collected data on gestational weight gain and mother and infant health outcomes, exploring associations between the two, and whether timing of gestational weight gain was linked to outcomes. Women attended study visits to coincide with their antenatal appointments, and at four and nine months postpartum. Of the total sample recruited ( $n=1035) ; 98 \%(n=1018)$ delivered a live-born baby, 494 returned for study assessments at four months postpartum and 328 returned at nine months postpartum (Mullaney et al., 2016a). Women were recruited opportunistically for the telephone interviews at the end of the BCIP study, during the last stages of follow-up assessments between November and December 2014. Women attending their final postpartum study visit during this period were provided with information about the telephone interviews. Interested women provided their contact details to the researcher. The women were then contacted to discuss the follow up interview and an interview date and time was arranged with those who wished to take part.

\section{Data collection}

Semi-structured telephone interviews were used as the method of data collection as they enabled an in depth account to be made by participants about their beliefs or perceptions on a particular topic, and allowed exploration of issues that arose during the interview. Telephone interviews were utilised in order to allow scheduling of 
discussions at the participants' convenience. The semi-structured nature of the interviews allowed flexibility for participants to voice their own ideas, whilst ensuring the discussion covered key topics relevant to the research aims. Open questions with additional probes were used, in order to ensure in-depth responses and data saturation.

Topic guides were developed following a review of the literature and discussion amongst the research team. They were designed to elicit participant's experiences of and opinions towards being weighed during pregnancy, and to weight management during pregnancy. The interviews were audio recorded and transcribed verbatim; transcripts were anonymised. The interviews lasted between 10 and 24 minutes.

\section{Data analysis}

The transcripts were analysed using a thematic analysis framework, which allows the identification and analysis of patterns across a dataset (Braun and Clarke, 2006). This began with reading the transcripts several times to ensure familiarity with the data. Transcripts were then coded, capturing data relevant to the research questions, and data extracts captured for each code. The codes were then reviewed to identify patterns which formed preliminary themes. Themes were checked against coded data extracts and the wider data set, and further refined. Finally, theme descriptions and names were compiled.

\section{Rigour}

The interviews were conducted by one researcher, who had previous experience and training in undertaking qualitative interviews, and with use of a topic guide, to ensure consistency across data collection. Two researchers reviewed the transcripts and analysis to ensure themes were agreed to be representative. 


\section{Ethical considerations}

The study was approved by the Coombe Women and Infants Hospital Research Ethics Committee and Dublin Institute of Technology Research Ethics. Participants provided verbal consent on the phone prior to the interview commencing. Transcripts were anonymised, and contact details were password protected. Participants were given pseudonyms for reporting quotes in the analysis.

\section{Results}

\section{Demographics}

Of the twenty women who provided their contact details at the final postpartum BCIP visit, ten took part in the telephone interview. The average age of the participants was 34.5 years; most had been weighed between two and four times during their pregnancies. One participant reported being regularly weighed at her GP surgery, and one participant was recorded as being weighed once during early pregnancy and twice postpartum. The average BMI of the group was $24.2 \mathrm{~kg} / \mathrm{m}^{2}$; eight participants were in the healthy weight range and two were in the overweight range.

\section{Qualitative findings}

Saturation was deemed to be reached after the tenth interview as no new themes were emerging. This paper reports on the themes identified as relating to routine weighing and weight management in pregnancy; three themes on this topic area were identified. The first, Routine weighing should be offered and is beneficial to all women, reflects the participants' beliefs that routine weighing should be part of standard care, as it has several benefits. The second, Health professionals have a part to play in weight management and pregnancy, discusses the important role of 
midwives and doctors in providing appropriate information and advice on gestational weight gain. The third, The influence of knowledge and experience on gestational weight gain and healthy lifestyle behaviours, captures the role that knowledge and previous pregnancy experience had on lifestyle behaviours and experiences of gestational weight gain, and what this could mean for other pregnant women.

\section{Theme 1: Routine weighing should be offered and is beneficial to all women}

The women in this group were of the opinion that routine weighing should be part of standard antenatal care.

'...I just think that it should be mandatory. You still have to ask to be weighed. I think that you should be weighed and it should be monitored.' Laura

'I think it wouldn't be a bad idea to weigh people when they're pregnant. I think it would benefit people more so than not.' Claire

Indeed, Pauline expressed confusion as to why she was not weighed at every appointment, and did not understand the reasoning for recording a weight at the booking appointment.

'Yeah, because I thought the whole point of maybe the first weigh-in would have been to keep an eye on it or something but then if they didn't do it again I don't know what the point was really of the first weigh-in.' Pauline

However, a point was raised that some women may not feel comfortable being weighed and so it should be a choice rather than compulsory.

'I think it should be their choice. They should be asked, yeah... but because some people do feel uncomfortable being weighed and some people want to be weighed so I think it should be part of our choices.' Barbara 
One of the perceived benefits of routine weighing was the potential to raise awareness of important health messages during pregnancy. There was a sense that myths such as 'eating for two' were still prevalent within social networks, and something experienced on a personal level by some participants during their first pregnancies. Therefore weighing was seen as a method of raising awareness of pregnancy weight gain and dispelling this myth.

'I would prefer to be weighed. I think they should weigh pregnant women because...I mean in my first with [child's name] I did eat for two. I was like oh yeah, you're allowed to eat for two. I wasn't weighed. I didn't know how much I put on, whereas with my second pregnancy I put on less than two stone because I was more aware of it.' Laura

More broadly, it was perceived that some women might be unaware of the importance of healthy eating during pregnancy, and routine weighing could provide a route to increasing awareness by accompanying the practice with educational messages.

'It didn't have much effect on what I did because I was conscious of it anyway, but I figure if I hadn't been conscious, if I hadn't been someone who looked after my weight it might be a bit of an eye opener, just speaking to other women that I know that are never concerned about their weight, and then one girl gained four stone when she was pregnant and couldn't understand why and she didn't realise she was gaining as much because she never weighed herself or ever think about it.' Claire

Indeed, Bernie felt regular weighing by a health professional would act as a catalyst to change unhealthy eating behaviours.

'...if I'm not made aware of okay, you're on the one side of unhealthy weight then I want to do something about it, whereas if I just leave it to myself I'm just sort of "oh l'll be fine. I'll 
give it another month and then l'll try." So I found it helpful yes. I personally found it helpful as a catalyst to try and sort of do something about it.' Bernie

For many participants, routine weighing served to provide information about their pregnancy, and answered their curiosity about their changing bodies.

'I didn't mind it, to be honest. In a way I found it interesting to be weighed when pregnant to see how your body is changing.' Shannon

'I like it because it was interesting just to find out, starting off, your weight and then how you were getting on with it, so from then when you had the baby then to see how much... did you lose weight or did you stay the same. So it was interesting for me.' Pauline

Women were interested in monitoring health parameters such as BMI, and general tracking of measurements, as well as comparing to previous pregnancies. There was a sense that monitoring their weight in this way provided reassurance.

'The second time round I had problems with my ligaments and stuff and I couldn't do as much exercise and that so it was interesting to know how much weight I was putting on compared to my first pregnancy where I had no problem.' Claire

'I think for their own benefit and if people do monitor their weight it's a good way of keeping a check on it and see that their BMI is healthy for their height and stuff like that. It is interesting to see as well. I just think that that would be a normal part of your appointments at the hospital. I think that would just be a normal thing.' Pauline

There was a focus in the interviews on the impact of pregnancy weight gain on the amount of weight women had left to lose after delivery, and therefore weighing was viewed as a useful tool to minimise potential postpartum weight retention. 
'And I suppose it helps then with afterwards too, if you are putting on too much weight that you don't have as hard a job afterwards.' Shannon

'I think it would be good because there is people that aren't conscious of their weight and have put on a lot of weight and then it's afterwards they go "oh why did that happen?" and find it really hard to lose.' Claire

The importance of returning to a healthy weight following delivery in preparation for future pregnancies was also raised by Bernie, who became aware of the risk of weight gain between pregnancies through a friend.

“...And she said my doctor just told me you better lose the weight because if you don't lose the weight and you're planning on having more kids you're going for heart disease, you're going for diabetes, you're going for all these types of... I said jeez, I didn't realise it could have that detrimental effect on your health if you kept on putting on the weight. I thought that was quite... it may have seemed mean to a pregnant woman, to say that, but she took it on board and she went for it, and I was like if someone had said that to me, that you're at risk of all these factors if you don't lose this weight before your next..." Bernie

Monitoring pregnancy weight gain, and in turn postpartum weight retention, was considered important to ensure good health for future pregnancies.

Theme 2: Health professionals have a part to play in weight management and pregnancy 
Participants reported that they received no advice on gestational weight gain during their standard antenatal care appointments, and felt that there was not enough advice available, only being given leaflets on healthy lifestyle.

“...my experience both times of being pregnant, I went semi private but even then the appointments are so short and so quick and the hospitals don't seem to have the time nor the staff to pay that much attention to detail..." Claire

"...There should be more information than one little booklet on the food pyramid." Claire

It was felt that health professionals did not have the time to communicate information about weight management and a healthy lifestyle during pregnancy, even in Claire's case where she had semi-private care. Participants felt that midwives should be better informed about weight management in pregnancy, as they were ideally placed to communicate the information and were perceived as an expert authority.

“You can self-monitor but l'm not a doctor or midwife so I don't know what's healthy or what's not..." Claire

“Yeah, because sometimes when you're given something on paper you tend to go by it. You know, the doctor hands you a piece of paper and goes these are good things, I think you might take it in more...So if there was information given I think people would be more conscious or read more about it or more informed." Emma

"No, I think they should... I know they give you leaflets but you get so many leaflets when you're pregnant you don't read them all. If the midwife was even more informed about weight and... for her to be able to give you information about it verbally and not just give you loads of leaflets and say go off and read them." Laura 
It was perceived that women would be more likely to act upon information provided by a midwife or other health professional, whereas they might not if they had to research the information themselves. Indeed, guidance on gestational weight gain from their healthcare team was wanted by this participant group.

\section{Theme 3: The influence of knowledge and experience on gestational weight gain and healthy lifestyle behaviours}

The majority of participants reported researching appropriate amounts of gestational weight gain online, using websites such as Baby Centre. The participants had used this information to determine how much weight they thought they should gain, alongside using experiences of past pregnancies.

"...I had read all the websites and what they recommend and that so I knew anything between two, two and a half stone would be for my height, because my starting off weight would be the amount that you should really put on when you're pregnant" Claire

"I read all the books and they said usually people put up a stone and a half to two stone, or whatever it was at the time, and I used to say God, do you know...l'd done all my research so I never really had overly much questions." Emma

"...when I was pregnant with the twins there was a US website I think the baby centre and you could put in your weight and your height and then what your...there was a range of what you should gain for each month, and I found that very useful because it went on your BMI. So it wasn't saying okay, you're way too heavy now at this stage. It's based on what you 
were before so I think something like that where it's like you're gaining a healthy..." Jacqueline

There was the perception that women used pregnancy as an excuse to overeat, which was considered a negative behaviour. Some participants commented that people don't know what healthy eating entailed, and were misinformed.

“...I would say with weight gain during pregnancy that it would be beneficial for people to have more of an understanding of health and what they're eating as well. Because I would be into healthy eating I still find it astonishing that people would think I'm eating healthy because I've had a baguette with salad and coleslaw and ham and cheese, when it's not, it's a lot of calories and it's a lot of food but people do think that's a healthy lunch. So I think weighing with a bit of health education would be good during pregnancy." Claire

"I honestly do believe, I think some women use it as an excuse, being pregnant, and they eat and eat and eat. I know girls, friends of mine that have done that and it's...I don't want to say disgusting but they just use it as an excuse, and there's no need. 'I'm pregnant; I can have three bars of chocolate instead of one.' And l've seen that, l've heard them, I sat with them, and I think that can't be good for you." Emma

Furthermore, there was a perception that some women did not know what the consequences of gestational weight gain were to themselves and their babies.

"Yes, I definitely think so, yes, because there's some women, like my sisters, that would just think it's okay to put on as much weight as you want during your pregnancies and not realising that you are doing yourself harm and afterwards the toll on your body how hard it is to lose it." Bernie 
This group of mothers considered themselves to be well informed and knowledgeable about gestational weight gain and a healthy lifestyle, yet felt that many women had low knowledge levels on these topics. There was a sense that if women had the correct information, they would be more likely to have a healthier approach to their pregnancies.

The majority of the participants interviewed reported a healthy prepregnancy diet and were motivated to manage their weight, and expected these health behaviours to continue through to pregnancy. Experiences during the prepregnancy period influenced expectations, with Kate expressing concern she wouldn't put enough weight on as this was something she usually had to monitor.

"I went to Weight Watchers, and I was a golden member in Weight Watchers so I'd go every few months. I would always have been conscious, so even if my clothes are tight or that I might need to lose a few pounds or do a bit of exercise, that kind of thing." Jacqueline

"I used to eat like I normally ate, I didn't eat more than I should have. I was just myself...I didn't change anything. I just kept going with what I do." Emma

"It wasn't a worry. I suppose it was in the sense that sometimes if I hadn't gained weight within a few weeks of being pregnant I thought oh gosh, is there something wrong?" Kate

First time mothers were unsure of what to expect in terms of their changing body shape and pregnancy weight gain, feeling they might 'balloon', yet were surprised when weight gain was predominately centred on their 'bump'. 
"I assumed that everything would balloon and everything would get very big, but it didn't. It was mainly confined to my belly. I thought my hips would gain lots of weight but they didn't, and my legs, I thought they would be very heavy but they weren't." Kate

Second time mothers discussed the different perspective on weight gain during the second pregnancy, as they reported the attitude of eating whatever they liked during their first pregnancy and subsequently found it difficult to lose the weight postpartum.

“It was that and because of...With my first I couldn't shift the weight for almost a year after. I found it very hard, because I did eat for two, because on your first you think everyone does it. "Oh you're allowed to eat to two; you can eat what you like." But that's not true; you can't eat what you like, unfortunately." Laura

Participants demonstrated an understanding of the link between weight and future health risks, in discussing the need to manage their weight in anticipation of future pregnancies. This was extended to healthy lifestyle behaviours, with several participants reflecting they would increase their fitness if they were to have another baby, to cope with the demands of pregnancy and labour.

\footnotetext{
"After a while then, after you get into the swing of things it's like...for me, I always try to maintain a certain healthy weight so for me it was important to try not to become too overweight, because then you were wanting to get pregnant again and so as not to snowball." Bernie
}

"I think l'd try to make myself a bit more fitter, because l'd imagine when you're pregnant the second time round you're going to be running after a toddler, you're going to have to try to keep up with everything and you've a lot more to do, so I think l'd try to get fitter." Emma 


\section{Discussion}

In this study, we explored the opinions and experiences of postnatal women who had been weighed throughout pregnancy, toward routine antenatal weighing and weight management during pregnancy.

The first theme, Routine weighing should be offered and is beneficial to all women, reflected that women expect to be routinely weighed throughout their pregnancies and did not experience anxiety as a result of being weighed. Furthermore, they perceived there would be several benefits to the practice, such as providing reassurance and minimising postpartum weight retention. These findings lend support to the idea that re-introducing the practice to antenatal care is acceptable to pregnant women. One of the reasons routine weighing of women was stopped in the 1990s was owing to the proposition that it caused unnecessary anxiety in women. The findings of this study and that of Daley et al. (2015) and Brownfoot et al. (2016) do not support this as a potential barrier to reintroducing the practice.

The second theme, Health professionals have a part to play in weight management and pregnancy, suggests that weighing women during antenatal care would create the opportunity to have a conversation about weight management with a health professional. Participants felt that there is a lack of information on gestational weight gain and a healthy lifestyle from healthcare professionals, which has been found in other qualitative studies (Arden et al. 2014; Atkinson et al. 2013; Brown and Avery, 2012; Olander et al. 2011). Therefore, this highlights the potential of routine weighing to provide a 'way in' to raise the issue of gestational weight gain. However it is important to consider how health professionals would feel about this, including consideration of their training and support needs (Basu et al. 2014; Heslehurst et al. 
2013; Heslehurst et al. 2014). Numerous qualitative studies have highlighted that health professionals consider weight a sensitive topic to raise and risk offending women, yet acknowledge that this is part of their role and women need to be informed about the consequences of excess gestational weight gain (Schmied et al. 2011; Singleton and Furber, 2014). Indeed, health professionals have suggested that weighing all women would avoid stigmatising just those who are overweight or obese (Knight-Agarwal et al. 2014), and would normalise the practice (Heslehurst et al. 2011). These findings suggest health professionals may be open to discussing gestational weight gain and routine weighing during pregnancy may provide a 'wayin' to raising the issue.

Finally, The influence of knowledge and experience on gestational weight gain and healthy lifestyle behaviours, indicated that women are already seeking out information on gestational weight gain from sources such as the internet, supporting findings from other qualitative studies with pregnant women (Olander et al. 2011), which reinforces the need to establish UK specific gestational weight gain guidelines. Furthermore, the discussions captured in this theme suggest myths such as 'eating for two' are still prevalent and perhaps encouraged by family members, which was similarly found by Brownfoot et al. (2016). The perception in this group that women still use pregnancy as an 'excuse' to overeat reinforces the need for consistent and timely advice on a healthy lifestyle in pregnancy. The women felt there was a lack of information on the consequences of excess GWG, information which is important to convey to women early on in pregnancy. However the best way to convey this information requires careful consideration, as women receive a significant amount of information at the booking appointment already and report that this is a lot to take process whilst also adjusting to being pregnant (Olander et al. 2011). 
This study supports previous findings that routinely weighing women during pregnancy does not cause anxiety (Daley et al. 2015; Brownfoot et al. 2016). Additionally, in line with Daley et al. (2015), regular weighing encouraged these women to be mindful of their gestational weight gain and associated health behaviours. This study expands on existing literature by considering the experiences and opinions of postnatal women; the in-depth accounts shared by the women in this sample provide insights into the first few months of the postpartum period as well as pregnancy, and suggests a potentially longer term impact of regular weighing. The women in this study felt regular weighing minimised the amount of weight they had to lose in the postpartum period as a result of being more conscious of their gestational weight gain. Furthermore, it adds to a body of research suggesting women want more information on gestational weight gain throughout antenatal care, and extends this by linking this desire for information to routine weighing; the women in this group felt weighing accompanied with educational messages from health professionals would be an effective way to promote healthy lifestyles during pregnancy.

\section{Strengths and limitations}

A strength of this research was the opportunity it provided to interview women who had been routinely weighed during their most recent pregnancy (less than one year postpartum), as routine weighing does not happen as part of standard care. Additionally, it reflected the views of women who had a normal BMI, whereas much of the literature focuses on participants who fall within an obese BMI category. Given proposals that routine weighing should be made a part of standard antenatal care for all women (Allen-Walker et al. 2016), it is important to consider the opinions of women from all BMl categories. 
It is important to acknowledge that this was a motivated sample taking part in research monitoring their pregnancy weight gain, which may reflect their positive attitudes to weighing. However the group articulated potential benefits of weighing which would apply to all women, which fits with existing findings that suggest health professionals find it a difficult topic to raise, and that women want more information about gestational weight gain. Although a strength in including women with a normal $\mathrm{BMI}$, it is possible that women with an overweight/ obese BMI may elicit different responses. On the other hand, existing research suggests overweight and obese pregnant women want more information (Atkinson et al. 2013; Brown and Avery, 2012) and interpret gestational weight gain as unimportant if health professionals do not raise it (Brown and Avery, 2012; Olander et al. 2011). Nevertheless, in order to ensure all potential viewpoints are captured on this topic, researchers involved in studies or working in clinical settings which involve weighing women throughout pregnancy should take the opportunity to gather further data on this subject.

Furthermore, though a convenient method of data collection for participants, the use of telephone interviews prevented observation of non-verbal communication which may have enriched interpretation of the results.

\section{Conclusion}

This study provides evidence suggesting that pregnant women do not find routine weighing to cause anxiety, and can see several benefits to the practice. Even in this well-informed group, advice and information on gestational weight gain and a healthy lifestyle in pregnancy was seen as lacking, yet information was considered important in order to ensure appropriate gestational weight gain. Feasibility studies into 
reintroducing routine weighing in antenatal care will provide further information on factors to consider, in order to address NICE recommendations on identifying appropriate gestational weight gain ranges for the UK, and ultimately improve outcomes for mothers and their children.

\section{References}

Allen-Walker, V., Woodside, J., Holmes, V., Young, I., Cupples, M.E., Hunter, A. \& McKinley, M.C., 2016. Routine weighing of women during pregnancy-is it time to change current practice? BJOG : an international journal of obstetrics and gynaecology, vol. 123 , no. 6 , pp. 871-874.

Arden, M.A., Duxbury, A.M. \& Soltani, H., 2014. Responses to gestational weight management guidance: a thematic analysis of comments made by women in online parenting forums. BMC pregnancy and childbirth, vol. 14, no. 1, pp. 216.

Atkinson, L., Olander, E.K. \& French, D.P., 2013. Why don't many obese pregnant and post-natal women engage with a weight management service? Journal of Reproductive and Infant Psychology, vol. 31, no. 3, pp. 245-256.

Basu, A., Kennedy, L., Tocque, K. \& Jones, S., 2014. Eating for 1, Healthy and Active for 2; feasibility of delivering novel, compact training for midwives to build knowledge and confidence in giving nutrition, physical activity and weight management advice during pregnancy. BMC pregnancy and childbirth, vol. 14, pp. 218-2393-14-218.

Braun, V. \& Clarke, V., 2006. Using thematic analysis in psychology. Qualitative research in psychology, vol. 3, no. 2, pp. 77-101.

Brown, A. \& Avery, A., 2012. Healthy weight management during pregnancy: what advice and information is being provided. Journal of Human Nutrition and Dietetics, vol. 25, no. 4, pp. 378-387.

Brownfoot, F.C., Davey, M.A. \& Kornman, L., 2016. Women's opinions on being weighed at routine antenatal visits. BJOG : an international journal of obstetrics and gynaecology, vol. 123, no. 2, pp. 263-270.

Daley, A.J., Jolly, K., Jebb, S.A., Lewis, A.L., Clifford, S., Roalfe, A.K., Kenyon, S. \& Aveyard, P., 2015. Feasibility and acceptability of regular weighing, setting weight gain limits and providing feedback by community midwives to prevent excess weight gain during pregnancy: randomised controlled trial and qualitative study. BMC obesity, vol. 2, pp. 35-015-0061-5. eCollection 2015. 
Dawes, M. \& Grudzinskas, J., 1991. Repeated measurement of maternal weight during pregnancy. Is this a useful practice? BJOG: An International Journal of Obstetrics \& Gynaecology, vol. 98, no. 2, pp. 189-194.

Fraser, A., Tilling, K., Macdonald-Wallis, C., Sattar, N., Brion, M., Benfield, L., Ness, A., Deanfield, J., Hingorani, A. \& Nelson, S.M., 2010. Association of maternal weight gain in pregnancy with offspring obesity and metabolic and vascular traits in childhood. Circulation, vol. 121, no. 23, pp. 2557-2564.

Heslehurst, N., Lang, R., Rankin, J., Wilkinson, J. \& Summerbell, C., 2007. Obesity in pregnancy: a study of the impact of maternal obesity on NHS maternity services. BJOG: An International Journal of Obstetrics \& Gynaecology, vol. 114, no. 3, pp. 334-342.

Heslehurst, N., Moore, H., Rankin, J., Ells, L.J., Wilkinson, J.R. \& Summberbell, C.D., 2011. How can maternity services be developed to effectively address maternal obesity? A qualitative study. Midwifery, vol. 27, no. 5, pp. e170-e177.

Heslehurst, N., Crowe, L., Robalino, S., Sniehotta, F.F., McColl, E. \& Rankin, J., 2014. Interventions to change maternity healthcare professionals' behaviours to promote weight-related support for obese pregnant women: a systematic review. Implementation science : IS, vol. 9, pp. 97-014-0097-9.

Heslehurst, N., Russell, S., McCormack, S., Sedgewick, G., Bell, R. \& Rankin, J., 2013. Midwives perspectives of their training and education requirements in maternal obesity: a qualitative study. Midwifery, vol. 29, no. 7, pp. 736-744.

Knight-Agarwal, C.R., Kaur, M., Williams, L.T., Davey, R. \& Davis, D., 2014. The views and attitudes of health professionals providing antenatal care to women with a high BMI: A qualitative research study. Women and Birth, vol. 27, no. 2, pp. 138-144.

Leslie, W., Gibson, A. \& Hankey, C., 2013. Prevention and management of excessive gestational weight gain: a survey of overweight and obese pregnant women. BMC pregnancy and childbirth, vol. 13, no. 1, pp. 10.

Modder, J. \& Fitzsimons, K., 2010. CMACE/RCOG Joint Guideline: management of women with obesity in pregnancy, Centre for Maternal and Child Enquiries and the Royal College of Obstetricians and Gynaecologists.

Mullaney, L., O'Higgins, A., Cawley, S., Daly, N., McCartney, D., \& Turner, M. J. (2016a). Weight and body composition trajectories between early pregnancy and four and nine months postpartum. Proceedings of the Nutrition Society, 75(E7) doi:10.1017/S0029665115004401

Mullaney, L., O' Higgins, A.C., Cawley, S., Kennedy, R., McCartney, D., \& Turner, M. J. (2016b). Breast-feeding and postpartum maternal weight trajectories. Public Health Nutrition, 19(8), 1397-1404. doi:10.1017/S1368980015002967 
National Institute for Clinical Excellence, 2010. NICE Public Health Guidance, No 27. Dietary Interventions and Physical Activity Interventions for Weight Management before, during and after Pregnancy. National Institute for Clinical Excellence.

Olander, E.K., Atkinson, L., Edmunds, J.K. \& French, D.P., 2011. The views of preand post-natal women and health professionals regarding gestational weight gain: An exploratory study. Sexual \& Reproductive Healthcare, vol. 2, no. 1, pp. 43-48.

Schmied, V.A., Duff, M., Dahlen, H.G., Mills, A.E. \& Kolt, G.S., 2011. 'Not waving but drowning': a study of the experiences and concerns of midwives and other health professionals caring for obese childbearing women. Midwifery, vol. 27, no. 4 , pp. $424-430$.

Scott-Pillai, R., Spence, D., Cardwell, C., Hunter, A. \& Holmes, V., 2013. The impact of body mass index on maternal and neonatal outcomes: a retrospective study in a UK obstetric population, 2004-2011. BJOG: An International Journal of Obstetrics \& Gynaecology, vol. 120, no. 8, pp. 932-9.

Singleton, G. \& Furber, C., 2014. The experiences of midwives when caring for obese women in labour, a qualitative study. Midwifery, vol. 30, no. 1, pp. 103111. 